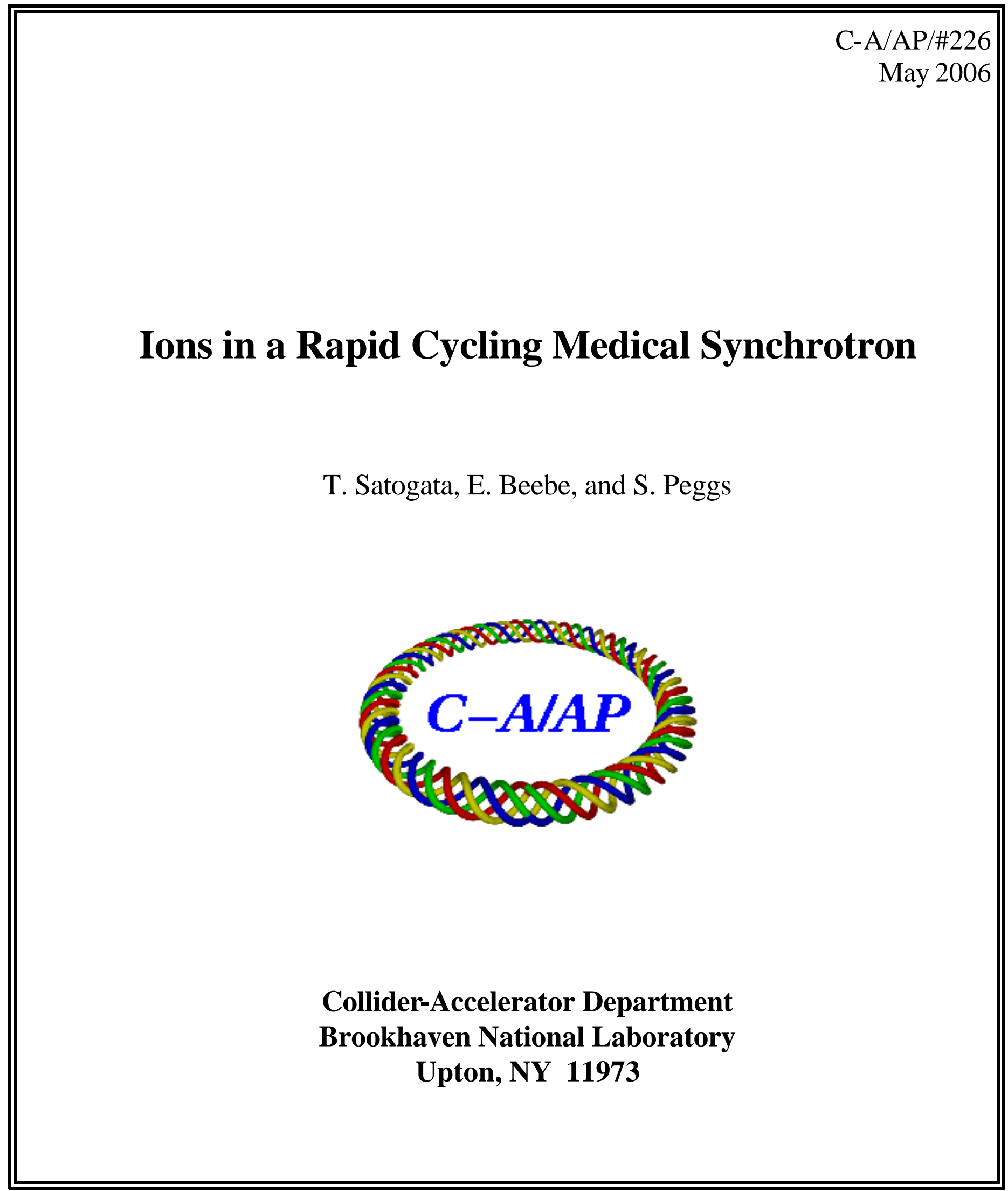


$\mathrm{CAD} / \mathrm{AP} / 226$

April 24, 2006

\title{
Ions in a Rapid Cycling Medical Synchrotron
}

\author{
T. Satogata, E. Beebe, and S. Peggs
}

\section{Introduction}

The Rapid Cycling Medical Synchrotron (RCMS) was originally designed for therapeutic irradiation of tumors with protons [1]. In contrast, this preliminary paper explores a design variant of the RCMS - the iRCMS - that is optimized for delivering ions with $Z / A \approx 0.5$ for therapy, biology, or physics.

\begin{tabular}{|c|c|c|c|c|c|c|c|c|c|}
\hline Species & $Z$ & $A$ & $Z / A$ & $\begin{array}{c}\text { Mass } \\
{[\mathrm{u}]}\end{array}$ & $\begin{array}{c}\text { Mass } / A \\
{[\mathrm{u}]}\end{array}$ & $\begin{array}{c}\text { Injection } \\
\text { K.E. } \\
{[\mathrm{MeV} / \mathrm{u}]}\end{array}$ & $\begin{array}{c}\text { Extraction } \\
(\max ) \mathrm{K} . \mathrm{E} . \\
{[\mathrm{MeV} / \mathrm{u}]}\end{array}$ & $\begin{array}{l}\text { Repetition } \\
\text { rate } \\
{[\mathrm{Hz}]}\end{array}$ & $\begin{array}{l}\text { Particles } \\
\text { per pulse }\end{array}$ \\
\hline \multicolumn{10}{|l|}{ Therapy } \\
\hline Protons & 1 & 1 & 1.000 & 1.0078 & & $(7)$ & $(250)$ & (30) & $\left(1.7 \times 10^{9}\right)$ \\
\hline Carbon & 6 & 12 & 0.500 & 12.0000 & 1.0000 & 20 & 450 & 30 & $2.7 \times 10^{7}$ \\
\hline \multicolumn{10}{|l|}{ Research } \\
\hline Helium & 2 & 4 & 0.500 & 4.0026 & 1.0007 & 20 & 450 & 30 & $1.6 \times 10^{8}$ \\
\hline Oxygen & 8 & 16 & 0.500 & 15.9949 & 0.9997 & 20 & 450 & 30 & $3.3 \times 10^{6}$ \\
\hline Neon & 10 & 20 & 0.500 & 19.9924 & 0.9996 & 20 & 450 & 30 & $3.3 \times 10^{6}$ \\
\hline Krypton & 36 & 84 & 0.428 & 83.9115 & 0.9989 & 17.1 & 385 & $\mathrm{o}(1 \mathrm{~Hz})$ & $3.3 \times 10^{6}$ \\
\hline Xenon & 54 & 131 & 0.412 & 130.9051 & 0.9993 & 16.5 & 371 & $\mathrm{o}(1 \mathrm{~Hz})$ & $3.3 \times 10^{6}$ \\
\hline
\end{tabular}

Table 1: Fundamental parameters of the iRCMS, an ion variant of the RCMS, designed to accelerate ions with $Z / A \approx 0.5$. Proton parameters from the RCMS are included for comparison purposes only, as their acceleration is best performed in a separate dedicated ring.

For the sake of simplicity, this paper assumes that the total mass of an ion is just

$$
M_{i o n}=A u
$$

Table 1 indicates that Equation 1 is accurate within an error of only about $\pm 0.1 \%$ for species from Helium through Xenon [2]. After making this assumption, all ion species with the same $Z / A$ value behave identically from the perspective of fully stripped single particle dynamics. For example, Table 1 shows that the maximum kinetic energy at extraction is the same for Helium, Oxygen and Neon (but is somewhat lower for Krypton and Xenon). Thus, it is natural to design the iRCMS for $Z / A=0.5$ operation, typically with carbon ions.

Table 2 compares the primary parameters of the carbon iRCMS with those of the proton RCMS. The iRCMS circumference of $\sim 55 \mathrm{~m}$ is about twice that of the RCMS. This is an approximate value, assuming only slightly longer straight sections, but scaling the arc magnets to new lengths for a higher rigidity with the same maximum bending field. Table 3 compares the basic kinematic parameters at injection and at the top of the energy ramp, for RCMS and iRCMS. 


\begin{tabular}{|lccc|}
\hline Accelerator & & RCMS & iRCMS \\
& & & \\
Charge to mass number ratio, $Z / A$ & & 1.0 & 0.5 \\
Kinetic energy (max) & {$[\mathrm{MeV} / \mathrm{u}]$} & 250 & 450 \\
& & & \\
Rigidity (max) & {$[\mathrm{Tm}]$} & 2.432 & 6.826 \\
Arc magnet dipole field (max) & {$[\mathrm{T}]$} & 1.436 & 1.432 \\
Arc magnet bending radius, $\rho$ & {$[\mathrm{m}]$} & 1.694 & 4.767 \\
& & & \\
Arc magnet magnetic length & {$[\mathrm{m}]$} & 0.760 & $\sim 2.14$ \\
Quadrupole magnetic length & {$[\mathrm{m}]$} & 0.14 & $\sim 0.39$ \\
Arc half-cell length & {$[\mathrm{m}]$} & 1.1 & $\sim 3.09$ \\
Number of half cells per arc & & 7 & 7 \\
Circumference, $C$ & {$[\mathrm{~m}]$} & 30.65 & $\sim 55$ \\
& & & \\
\hline
\end{tabular}

Table 2: Preliminary primary physical parameters of the RCMS and the iRCMS.

\begin{tabular}{|lccccc|}
\hline & & \multicolumn{2}{c}{ RCMS } & \multicolumn{2}{c|}{ iRCMS } \\
& & injection & extraction & injection & extraction \\
Kinetic energy, $K$ & {$[\mathrm{MeV} / \mathrm{u}]$} & 7 & 250 & 20 & 450 \\
Momentum, $p$ & {$[\mathrm{MeV} / \mathrm{uc}]$} & 114.8 & 729.1 & 194.8 & 1023.2 \\
Lorentz $\gamma$ & & 1.0075 & 1.2664 & 1.0213 & 1.4796 \\
Lorentz $\beta$ & & 0.122 & 0.614 & 0.203 & 0.737 \\
& & & & & \\
Rigidity, $B \rho$ & {$[\mathrm{Tm}]$} & 0.383 & 2.432 & 1.299 & 6.826 \\
Dipole field, $B$ & {$[\mathrm{~T}]$} & 0.226 & 1.436 & 0.272 & 1.432 \\
Revolution frequency, $F_{\text {rev }}$ & {$[\mathrm{MHz}]$} & 1.188 & 6.002 & 1.108 & 4.018 \\
Revolution period, $T_{\text {rev }}$ & {$[\mu \mathrm{s}]$} & 0.842 & 0.166 & 0.903 & 0.249 \\
& & & & & \\
\hline
\end{tabular}

Table 3: Kinematic parameters at injection and at the maximum extraction energy at the top of the acceleration ramp, for RCMS (protons) and iRCMS (carbon ions). 


\section{Preliminary Design Comments}

A comparison of beam and accelerator parameters through the acceleration ramp is plotted in Figures 1 and 2, with numerical values listed in Table 6.

The iRCMS Radio Frequency (RF) range is only slightly lower than for the RCMS, because the competing effects of increased circumference and increased speed almost balance. With $Z / A=1 / 2$, iRCMS requires approximately twice the RF voltage of the RCMS. The original RCMS lattice includes a space reserved for a second RF cavity [1], so additional straight section space is not required to support this higher RF voltage. It seems that the same RF technology used for the RCMS can also be used for the iRCMS.

The same maximum bending field of $1.43 \mathrm{~T}$ is assumed for the iRCMS main arc magnets, in order to maintain simplicity of design, lower costs, and to leverage the existing RCMS design. The dynamic range of the field is somewhat lower for iRCMS because the momentum range is smaller than for RCMS. The field ramp rate $d B / d t$ is correspondingly smaller for the same $30 \mathrm{~Hz}$ repetition rate, as are eddy current effects. Thus, at first glance, magnet issues seem similar for RCMS and iRCMS. Nonetheless, a detailed analysis of eddy current effects requires a detailed magnet, vacuum chamber and lattice design.

Detailed lattice design and optimization is also required to maintain aperture constraints and to match to dispersion-free straights. Simply scaling the lengths of the main magnets and arcs with the rigidity is reasonable in this preliminary context, in order to evaluate iRCMS feasibility, but arc beam size constraints will require (presumably modest) changes in their design parameters. These changes are not expected to modify the fundamental observation that an iRCMS with listed parameters appears feasible with RCMS magnet and RF technology.

\section{Injector Considerations}

The iRCMS injector has different challenges than the RCMS injector. It must provide the fully stripped ions listed in Table 1 at $20 \mathrm{MeV}$, and should be able to change between ion species quickly, with little contamination from other ion species with similar $Z / A$.

Electron Beam Ion Trap (EBIT) and Electron Beam Ion Source (EBIS) technologies are the most promising for an iRCMS injector. When used to produce extracted ions both source types are operated using the principles of the Electron Beam Ion Source described in [5].

In practice, the EBIT source usually operates with higher current densities and higher electron beam energies, allowing it to produces bare ions (i.e. all electrons stripped away) up to Uranium [6]. EBIT sources are traditionally short, with interaction regions 2.5-4.0 cm long, a design which is sufficient for experiments and observations with trapped ions. The EBIT operates with relatively low currents, up to $250 \mathrm{~mA}$ for low energy beams. For light ions one can expect $\sim 10^{7}$ ions/pulse and confinement periods of less than $30 \mathrm{~ms}$. However, for the production of highly charged heavy ions such as $\mathrm{Xe}^{54+}$, the $73 \mathrm{keV}$ e-beam current is limited to $70 \mathrm{~mA}$, and only about $500 \mathrm{Xe}^{54+}$ ions/pulse are extracted after a $500 \mathrm{~ms}$ confinement period[6].

EBIS sources are designed to provide beams for accelerators and external experiments, and hence typically have an ionization region about $1 \mathrm{~m}$ long. These sources typically operate with electron beam with $I_{e}<0.5 \mathrm{~A}, E_{e}<30 \mathrm{keV}$ and electron beam densities ranging from 100-2000 A/ $\mathrm{cm}^{2}$. High current EBIS technology under development at BNL uses a $20-30 \mathrm{keV}, 10 \mathrm{~A}, 575 \mathrm{~A} / \mathrm{cm}^{2}$ electron beam to produce extracted ion beams up to $2.5 \times 10^{11}$ charges per 10-40 microsecond pulse. This EBIS is designed to provide partially-stripped high-brightness ion beams up to $\mathrm{U}^{45+}$, with a 1 second switching time between species, $5 \mathrm{~Hz}$ rep rate, and $17 \mathrm{keV} / \mathrm{u}$ extraction energy [4].

The production of bare light ions up to Ne is relatively easy for both conventional EBIT and EBIS sources. The conventional EBIS "Dione" at Saclay routinely produced $6.7 \times 10^{8}$ ions/pulse of $\mathrm{C}^{6+}$ and $10^{8}$ ions/pulse of $\mathrm{Ne}^{10+}$ in less than $30 \mathrm{~ms}$, operating with a $1 \mathrm{~m}$ trap, and electron beam current of up to $0.5 \mathrm{~A}$, and current density of about $1000 \mathrm{~A} / \mathrm{cm}^{2}$ [7]. Some source development may be necessary if it is required to produce bare ions of the heavier research ions in quantities greater than $10^{6}$ ions/pulse in less than $30 \mathrm{~ms}$.

Bare ion production of a particular species depends on a threshold bombarding electron beam energy $E_{e}$, the current density $j_{e}$, and the confinement time $T$. An increased quantity of ion charge can be produced and trapped with increased electron beam space charge, which is proportional to the current $I_{e}$ and the trap length $L$ for a given energy. Donets has presented the relationship between $E_{e}$ and the ionization factor $j_{e} T$ [5]. The graph can be used to construct Table 4.

There is a strong inverse correlation between beam intensity and charge state with EBIS and EBIT. Higher intensities and repetition rates are only achievable with lower charge states from the injector. The column with the 


\begin{tabular}{|ccccccc|}
\hline $\begin{array}{c}\text { Ion } \\
\text { species }\end{array}$ & $\begin{array}{c}\text { Last electron } \\
\text { ionization potential }[\mathrm{V}]\end{array}$ & $\begin{array}{c}\text { Electron beam } \\
\text { energy }[\mathrm{eV}]\end{array}$ & $\begin{array}{c}\text { Ionization } \\
\text { factor }\left[\mathrm{C} / \mathrm{cm}^{2}\right]\end{array}$ & $\begin{array}{c}\text { Conf period } T[\mathrm{~ms}] \text { for } j_{e}\left[\mathrm{~A} / \mathrm{cm}^{2}\right] \\
j_{e}=1000\end{array}$ & $j_{e}=5000$ & $j_{e}=55000$ \\
$\mathrm{C}^{6+}$ & $\sim 490$ & 980 & $\sim 10$ & 10 & 2 & 0.2 \\
$\mathrm{O}^{8+}$ & $\sim 870$ & 1740 & $\sim 10$ & 10 & 2 & 0.2 \\
$\mathrm{Ne}^{10+}$ & $\sim 1360$ & 2720 & $\sim 32$ & 32 & 6 & 0.6 \\
$\mathrm{Fe}^{26+}$ & $\sim 9280$ & 18560 & $\sim 320$ & 320 & 64 & 6 \\
$\mathrm{Kr}^{36+}$ & $\sim 17950$ & 35900 & $\sim 5600$ & 5600 & 1120 & 101 \\
$\mathrm{Xe}^{54+}$ & $\sim 41300$ & 82600 & $\sim 32000$ & 32000 & 6400 & 582 \\
& & & & & & \\
\hline
\end{tabular}

Table 4: Ion species and properties for an iRCMS EBIS preinjector for bare ion production, including confinement period for various electron beam current densities $j_{e}$.

\begin{tabular}{|c|c|c|c|c|c|c|c|c|c|}
\hline \multirow[t]{2}{*}{ Species } & \multicolumn{2}{|c|}{$I_{e}$} & \multirow{2}{*}{$\begin{array}{c}\% \text { compensation } \\
\text { by bare ion } \\
\text { charge state }\end{array}$} & \multirow{2}{*}{$\begin{array}{c}\text { Ionization } \\
\text { potential } \\
{[\mathrm{kV}]}\end{array}$} & \multirow{2}{*}{$\begin{array}{c}\mathrm{e}^{-} \text {beam } \\
\text { energy } \\
{[\mathrm{keV}]}\end{array}$} & \multicolumn{2}{|c|}{ Ions/pulse } & \multicolumn{2}{|c|}{ Conf period $T$} \\
\hline & $\begin{array}{r}\text { EBIT } \\
{[\mathrm{mA}]}\end{array}$ & $\begin{array}{l}\text { EBIS } \\
{[\mathrm{mA}]}\end{array}$ & & & & EBIT & EBIS & $\begin{array}{c}\text { EBIT } \\
{[\mathrm{ms}]}\end{array}$ & $\begin{array}{c}\text { EBIS } \\
{[\mathrm{ms}]}\end{array}$ \\
\hline $\mathrm{He}^{2+}$ & 200 & 1000 & 20 & & 20 & $6.0 \times 10^{7}$ & $1.0 \times 10^{10}$ & & \\
\hline $\mathrm{C}^{6+}$ & 200 & 1000 & 20 & 0.49 & 20 & $2.0 \times 10^{7}$ & $1.7 \times 10^{9}$ & 2.5 & 2 \\
\hline $\mathrm{O}^{8+}$ & 200 & 1000 & 20 & 0.87 & 20 & $1.5 \times 10^{7}$ & $1.3 \times 10^{9}$ & 2.5 & 2 \\
\hline $\mathrm{Ne}^{10+}$ & 200 & 1000 & 20 & 1.36 & 20 & $1.2 \times 10^{7}$ & $1.1 \times 10^{9}$ & 8.0 & 6 \\
\hline $\mathrm{Fe}^{26+}$ & 150 & 1000 & 5 & 9.28 & 30 & $9.4 \times 10^{5}$ & $1.0 \times 10^{8}$ & & 64 \\
\hline $\mathrm{Kr}^{36+}$ & 100 & 1000 & 2 & 17.95 & 40 & $2.7 \times 10^{5}$ & $2.0 \times 10^{7}$ & 2800 & 1120 \\
\hline $\mathrm{Xe}^{54+}$ & 70 & 1000 & 1 & 41.30 & 73 & $2.0 \times 10^{4}$ & $7.0 \times 10^{6}$ & 22800 & 6400 \\
\hline
\end{tabular}

Table 5: Comparison of ion and preinjector parameters between example EBIT and EBIS preinjectors for iRCMS. The EBIT ions/pulse column assumes electron beam fluxes of $j_{e}=4000-1400 \mathrm{~A} / \mathrm{cm}^{2}$. The EBIS ions/pulse column assumes electron beam fluxes of $j_{e}=5000 \mathrm{~A} / \mathrm{cm}^{2}$.

heading 55,000 A/ $\mathrm{cm}^{2}$ in Table 4 is from a High Current EBIT source proposed by Marrs [8]. Such a source would be similar to the BNL EBIS in that it would operate with a $5 \mathrm{~A}$ electron beam and would have an extended length of $25 \mathrm{~cm}$. Another option would be to accept less frequent pulses for heavy ions and adapt the BNL EBIS to use a high energy $5000 \mathrm{~A} / \mathrm{cm}^{2}$ electron beam. Reducing the BNL EBIS electron beam from $10 \mathrm{~A}$ to $1 \mathrm{~A}$ should make it easier to raise the beam energy from $30 \mathrm{kV}$ to $80 \mathrm{kV}$ while still providing sufficient quantities of ions in the $0.7 \mathrm{~m}$ trap length. In Table 5 the number of ions/pulse of each species is estimated for a conventional EBIT [6] and a BNL EBIS adapted for high voltage, $5000 \mathrm{~A} / \mathrm{cm}^{2}$ operation. The quantities are believed to be conservative for all entries. The EBIS $\mathrm{Xe}^{54+}$ result is significantly larger than the EBIT experimentally obtained value because of a factor of 3.5 improvement in current density due to the electron beam current.

For heavy ions the current density of the electron beam determines how long it takes to convert all the ions into the fully stripped state. In general, unless there is strong cooling, some ions will be lost with increased confinement time. If more bare ions are available than required, the confinement time can be reduced such that ions are extracted before all of them have reached the fully stripped state. For example, by extracting the ions after only $10 \%$ of the time necessary to produce all bare ions, one could expect to have produced about $2 \%$ bare ions. This enables one to increase the pulse frequency at the expense of quantity of the bare ions extracted per pulse. One can see from the required EBIT confinement times that extracting $\mathrm{Xe}^{54+}$ after only $500 \mathrm{~ms}$ gives a relatively low abundance. The charge analyzed spectrum shows the most probable charge state to be $\mathrm{Xe}^{50+}[6]$. 


\section{Conclusions}

1. An ion variant of the RCMS, the iRCMS, accelerates ions from Helium to Neon over a kinetic energy range from 20 to $450 \mathrm{MeV} / \mathrm{u}$, and heavier ions such as Krypton and Xenon from about 17 to about $380 \mathrm{MeV} / \mathrm{u}$.

2. A simple scaling of main arc magnet lengths produces a machine circumference of approximately $55 \mathrm{~m}$, about twice that of the RCMS. Higher accuracy requires more realistic lattice design development.

3. Magnet and RF technologies suitable for the RCMS are also appropriate for iRCMS. No particular challenges are foreseen.

4. Ion generation appears to be the most challenging technical issue. EBIT and EBIS ion sources promise to deliver fully stripped high current light ion (Carbon) therapy beams, in combination with an RFQ and a DTL.

5. The generation of high intensity heavy ion beams (in a high charge-state) requires further development. Repetition rates for heavy ions will likely be a few $\mathrm{Hz}$ or less. This is probably adequate for research beams, but probably not for therapy beams.

\section{Acknowledgments}

We are very grateful for productive discussions with Michael Herman about sample user specifications for the iRCMS.

\section{References}

[1] Conceptual Design of the RCMS, C. Gardner and S. Peggs (editors), unpublished, March 27, 2003.

[2] The 2003 atomic mass evaluation, G. Audi, A.H. Wapstra and C. Thibault, Nuclear Physics A 729 (2003) pp. 337-676.

[3] Extraction of highly charged ions from the electron beam ion trap at LBNL for applications in surface analysis and materials science, T. Schenkel et al., Rev. Sci. Instr. 73 2, February 2002, pp. 663-6.

[4] Progress on Test EBIS and the Design of an EBIS-Based RHIC Preinjector, J.G. Alessi et al., 2005 Particle Accelerator Conference, pp. 363-5 (Knoxville, 2005).

[5] E.D. Donets in "The Physics and Technology of Ion Sources", edited by I.G.Brown, (Wiley,New York 1989), chap. 12 .

[6] J.W. McDonald et. al. RSI 73 (1) 2002 pp.30-35.

[7] J. Faure et. al. AIP conf proc. no.188, pp. 102-112.

[8] R.E. Marrs et. al. UCRL-JC-132602 (1998). 

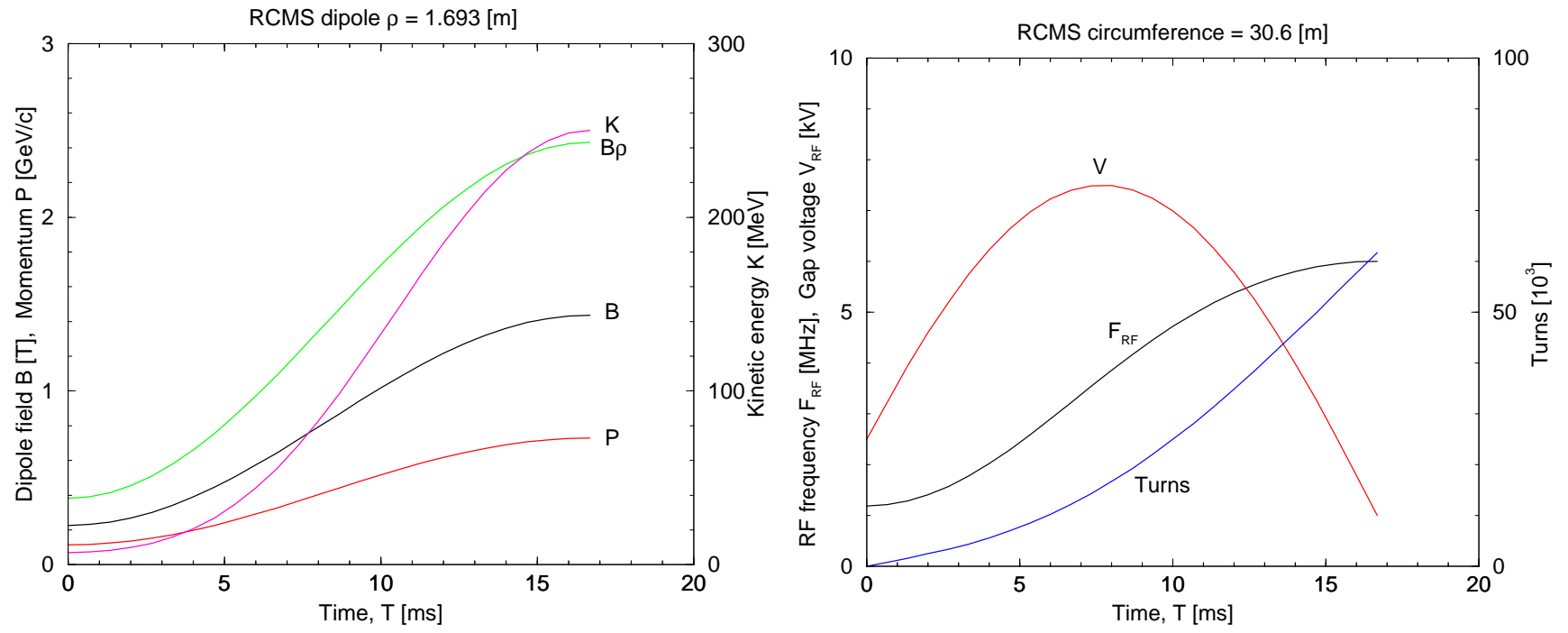

Figure 1: Key parameters in the RCMS acceleration cycle for protons. LEFT: dipole field $B$, rigidity $B \rho$, kinetic energy $K$, and momentum $P$, in the acceleration (rising) phase of a synchrotron cycle. RIGHT: RF frequency $F_{R F}$, $\mathrm{RF}$ gap voltage $V_{R F}$, and total number of turns, for the nominal proton RCMS acceleration ramp.
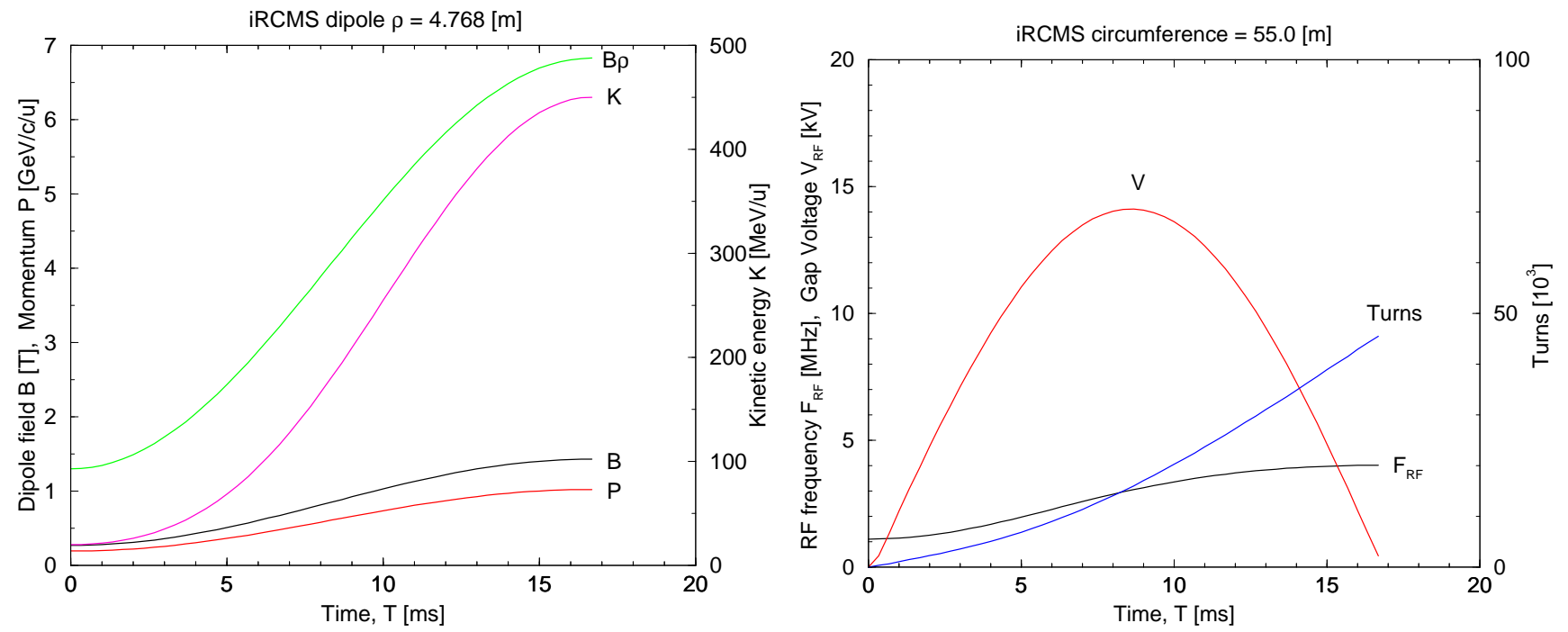

Figure 2: Key parameters in the iRCMS acceleration cycle for ions with $\mathrm{Z} / \mathrm{A}=0.5$. LEFT: dipole field $B$, rigidity $B \rho$, kinetic energy $K$, and momentum $P$, in the acceleration (rising) phase of a synchrotron cycle. RIGHT: RF frequency $F_{R F}$, RF gap voltage $V_{R F}$, and total number of turns, for the nominal $\mathrm{Z} / \mathrm{A}=0.5$ iRCMS acceleration ramp. 


\begin{tabular}{|c|c|c|c|c|c|c|c|c|c|c|}
\hline $\begin{array}{l}T \\
\mathrm{~ms}\end{array}$ & $\begin{array}{l}B \\
\mathrm{~T}\end{array}$ & $\begin{array}{c}\dot{B} \\
\mathrm{~T} / \mathrm{s}\end{array}$ & $\begin{array}{c}\dot{B} / B \\
\mathrm{~Hz}\end{array}$ & $\beta$ & $\gamma$ & $\begin{array}{c}K \\
\mathrm{MeV} / \mathrm{u}\end{array}$ & $\begin{array}{c}P \\
\mathrm{MeV} / \mathrm{c} / \mathrm{u}\end{array}$ & $\begin{array}{l}F_{\text {rev }} \\
\mathrm{MHz}\end{array}$ & Turns & $\begin{array}{l}V_{r f} \\
\mathrm{kV}\end{array}$ \\
\hline \multicolumn{11}{|c|}{ RCMS (protons) } \\
\hline 0.000 & 0.226 & 0.0 & 0.0 & 0.121 & 1.007 & 7.00 & 114.82 & 1.188 & 0 & 2.500 \\
\hline 1.000 & 0.237 & 21.4 & 90.2 & 0.127 & 1.008 & 7.68 & 120.27 & 1.244 & 1198 & 3.595 \\
\hline 2.000 & 0.269 & 42.0 & 156.3 & 0.144 & 1.011 & 9.86 & 136.39 & 1.407 & 2489 & 4.598 \\
\hline 3.000 & 0.320 & 61.1 & 190.7 & 0.171 & 1.015 & 13.99 & 162.64 & 1.671 & 3977 & 5.484 \\
\hline 4.000 & 0.390 & 78.1 & 200.1 & 0.207 & 1.022 & 20.68 & 198.07 & 2.020 & 5758 & 6.229 \\
\hline 5.000 & 0.476 & 92.3 & 194.0 & 0.249 & 1.033 & 30.57 & 241.44 & 2.438 & 7913 & 6.816 \\
\hline 6.000 & 0.574 & 103.2 & 179.9 & 0.296 & 1.047 & 44.15 & 291.20 & 2.899 & 10503 & 7.227 \\
\hline 7.000 & 0.681 & 110.5 & 162.3 & 0.346 & 1.066 & 61.62 & 345.59 & 3.381 & 13562 & 7.454 \\
\hline 8.000 & 0.793 & 113.8 & 143.5 & 0.394 & 1.088 & 82.76 & 402.69 & 3.858 & 17103 & 7.491 \\
\hline 9.000 & 0.907 & 113.1 & 124.7 & 0.441 & 1.114 & 106.90 & 460.48 & 4.309 & 21114 & 7.336 \\
\hline 10.000 & 1.018 & 108.5 & 106.5 & 0.483 & 1.142 & 132.96 & 516.89 & 4.720 & 25563 & 6.993 \\
\hline 11.000 & 1.123 & 99.9 & 89.0 & 0.519 & 1.170 & 159.54 & 569.95 & 5.078 & 30407 & 6.471 \\
\hline 12.000 & 1.217 & 87.9 & 72.2 & 0.550 & 1.197 & 185.11 & 617.77 & 5.379 & 35589 & 5.784 \\
\hline 13.000 & 1.297 & 72.7 & 56.0 & 0.575 & 1.222 & 208.10 & 658.65 & 5.620 & 41053 & 4.949 \\
\hline 14.000 & 1.361 & 54.9 & 40.4 & 0.593 & 1.242 & 227.07 & 691.14 & 5.801 & 46738 & 3.988 \\
\hline 15.000 & 1.407 & 35.2 & 25.1 & 0.606 & 1.257 & 240.84 & 714.10 & 5.924 & 52584 & 2.924 \\
\hline 16.000 & 1.431 & 14.3 & 10.0 & 0.612 & 1.265 & 248.52 & 726.71 & 5.990 & 58534 & 1.786 \\
\hline 16.667 & 1.436 & -0.0 & -0.0 & 0.614 & 1.266 & 250.00 & 729.13 & 6.002 & 62530 & 1.000 \\
\hline \multicolumn{11}{|c|}{ iRCMS (carbon ions) } \\
\hline 0.000 & 0.272 & 0.0 & 0.0 & 0.203 & 1.021 & 20.00 & 194.7 & 1.108 & 0 & 0 \\
\hline 1.000 & 0.283 & 20.5 & 72.4 & 0.211 & 1.023 & 21.52 & 202.1 & 1.148 & 1115 & 2.219 \\
\hline 2.000 & 0.313 & 40.2 & 128.4 & 0.232 & 1.028 & 26.33 & 223.8 & 1.265 & 2297 & 4.761 \\
\hline 3.000 & 0.363 & 58.5 & 161.4 & 0.266 & 1.037 & 35.16 & 259.2 & 1.452 & 3619 & 7.117 \\
\hline 4.000 & 0.430 & 74.8 & 174.1 & 0.311 & 1.052 & 48.96 & 307.0 & 1.695 & 5148 & 9.230 \\
\hline 5.000 & 0.511 & 88.4 & 172.8 & 0.363 & 1.073 & 68.68 & 365.5 & 1.979 & 6936 & 11.038 \\
\hline 6.000 & 0.605 & 98.8 & 163.3 & 0.419 & 1.101 & 94.93 & 432.6 & 2.282 & 9015 & 12.476 \\
\hline 7.000 & 0.708 & 105.8 & 149.5 & 0.475 & 1.136 & 127.73 & 505.9 & 2.587 & 11399 & 13.487 \\
\hline 8.000 & 0.816 & 109.0 & 133.7 & 0.528 & 1.177 & 166.36 & 582.9 & 2.877 & 14085 & 14.027 \\
\hline 9.000 & 0.925 & 108.4 & 117.2 & 0.576 & 1.223 & 209.39 & 660.9 & 3.139 & 17051 & 14.069 \\
\hline 10.000 & 1.031 & 103.9 & 100.8 & 0.618 & 1.272 & 254.83 & 736.9 & 3.367 & 20269 & 13.607 \\
\hline 11.000 & 1.131 & 95.7 & 84.6 & 0.653 & 1.320 & 300.31 & 808.5 & 3.558 & 23702 & 12.654 \\
\hline 12.000 & 1.221 & 84.2 & 68.9 & 0.681 & 1.366 & 343.33 & 873.0 & 3.713 & 27315 & 11.244 \\
\hline 13.000 & 1.299 & 69.6 & 53.6 & 0.703 & 1.407 & 381.50 & 928.1 & 3.833 & 31071 & 9.426 \\
\hline 14.000 & 1.360 & 52.6 & 38.7 & 0.719 & 1.440 & 412.68 & 971.9 & 3.922 & 34936 & 7.266 \\
\hline 15.000 & 1.403 & 33.8 & 24.1 & 0.730 & 1.464 & 435.13 & 1002.9 & 3.981 & 38879 & 4.841 \\
\hline 16.000 & 1.427 & 13.7 & 9.6 & 0.736 & 1.477 & 447.59 & 1019.9 & 4.012 & 42872 & 2.238 \\
\hline 16.667 & 1.432 & -0.0 & -0.0 & 0.737 & 1.480 & 450.00 & 1023.2 & 4.018 & 45548 & 0.450 \\
\hline
\end{tabular}

Table 6: The main accelerator and beam parameters during the acceleration phase of the synchrotron cycle for the RCMS with $\mathrm{Z} / \mathrm{A}=1$ protons (top), and iRCMS with $\mathrm{Z} / \mathrm{A}=0.5$ carbon ions (bottom). 